\title{
NOTE ON LINEARLY GOMPACT ABELIAN GROUPS
}

\author{
L. FUCHS ${ }^{1}$
}

(Received 29 August 1967)

By a group $A$ is meant throughout an additively written abelian group. $A$ is said to have a linear topology if there is a system of subgroups $U_{i}(i \in I)$ of $A$ such that, for any $a \in A$, the cosets $a+U_{i}(i \in I)$ form a fundamental system of neighborhoods of $a$. The group operations are continuous in any linear topology; the topologies are always assumed to be Hausdorff, that is, $\bigcap_{i} U_{i}=0$. A linearly compact group is a group $A$ with a linear topology such that if $a_{j}+A_{j}(j \in I)$ is a system of cosets modulo closed subgroups $A_{j}$ with the finite intersection property (i.e. any finite number of $a_{j}+A_{j}$ have a non-void intersection), then the intersection $\bigcap_{j}\left(a_{j}+A_{j}\right)$ of all of them is not empty.

Our present aim is to study the algebraic structure of linearly compact groups. They can be characterized as inverse limits of groups with minimum condition on subgroups. It turns out that the class of linearly compact groups lies between the classes of compact and algebraically compact groups: every group that admits a compact topology is linearly compact in some suitable topology, while all linearly compact groups are algebraically compact. A complete system of invariants can be obtained for linearly compact groups. In obtaining these invariants, a fundamental role is played by the fact that every linearly compact group $A$ can be decomposed as a direct product $A=\prod_{p} A_{p}$ where, for each prime $p, A_{p}$ is a topological module over the $p$-adic integers, with the product topology on $A$. For the components $A_{p}$ we can apply the duality theory of Kaplansky [10] and Schöneborn [15] (between discrete and linearly compact $p$-adic modules) in order to get a full structure theorem on linearly compact groups.

We shall use the following notations: $Z=$ infinite cyclic group; $Z(n)=$ cyclic group of finite order $n ; Z\left(p^{\infty}\right)=$ group of type $p^{\infty} ; Q=$ group of rational numbers; $R=$ group of real numbers; $K=$ real numbers $\bmod 1 ; J_{p}=$ group of $p$-adic integers; $K_{p}=$ group of $p$-adic numbers. The symbols $\oplus$ and $\Pi$ denote direct sums and products, respectively. GP-6862.

1 This work was supported by the National Science Foundation research grant number 
We shall need some elementary facts on linearly compact groups; for the proofs of the following we refer to Lefschetz [12].

(a) A subgroup of a linearly compact group is linearly compact if and only if it is closed.

(b) The image of a linearly compact group under a continuous homomorphism is again linearly compact.

(c) The product of linearly compact groups is linearly compact in the product topology.

(d) The inverse limit of linearly compact groups is linearly compact.

(e) Linearly compact groups are complete.

(f) A group which is compact in a linear topology is linearly compact.

The following lemma is due to Leptin [13] and Schöneborn [15].

Lemma 1. A group with the discrete topology is linearly compact if and only if it satisfies the minimum condition on subgroups.

Assume $A$ linearly compact in the discrete topology. Then $A$ does not contain elements of infinite order, for $Z$ is not linearly compact. (If $\pi=s_{0}+s_{1} p+s_{2} p^{2}+\cdots$ is a non-rational $p$-adic integer, then the closed cosets $s_{0}+s_{1} p+\cdots+s_{n-1} p^{n-1}+p^{n} Z$ have no element in common.) Thus $A$ is torsion; let $S$ be its socle. Choose a basis $\left\{b_{j}\right\}_{j \in I}$ for $S$ with each $b_{j}$ of prime order. Let $B_{i}$ be the subgroup generated by all $b_{j}$ with $j \neq i$. The cosets $b_{i}+B_{i}(i \in I)$ have the finite intersection property, but the intersection of all of them is empty unless $I$ is a finite index set. Hence $S$ is finite which is equivalent to the minimum condition on subgroups [16].

Conversely, if $A$ satisfies the minimum condition, and if the cosets $a_{i}+A_{i}(i \in I) \bmod$ subgroups $A_{i}$ have the finite intersection property, then selecting a minimal one among the finite intersections $A_{i_{1}} \cap \cdots \cap A_{i_{n}}$, it follows at once that the intersection of all $a_{i}+A_{i}$ is equal to the intersection of cosets corresponding to a minimal finite intersection, and so it is not empty.

COROLLARY 1. If $A$ is a linearly compact group and $U$ is an open subgroup of $A$, then $A / U$ satisfies the minimum condition.

In fact, $A / U$ is then linearly compact in the discrete topology.

Some of our subsequent theorems are based on the following result.

TheOREM 1. A group is linearly compact if and only if it is an inverse limit of groups with minimum condition. 
The 'if' part is an immediate consequence of Lemma $l$ and (d). To prove the 'only if' part, assume $A$ linearly compact, and let the subgroups $U_{i}(i \in I)$ form a fundamental system of neighborhoods about 0 . Without loss of generality, the system of the $U_{i}$ may be assumed closed under finite intersection. By Corollary $1, A / U_{i}$ satisfies the minimum condition, for every $i \in I$. The groups $A / U_{i}$ with the obvious maps $\pi_{i}^{3}: a+U_{i} \rightarrow a+U_{j}$ (for $U_{i} \subseteq U_{j}$ ) form an inverse system whose inverse limit must be $A$, since the image of $A$ under the natural map is a dense subgroup of the inverse limit, and by (a), the image must be the whole inverse limit.

From (e) it follows easily (cf. Leptin [13]):

Lemma 2. A group with a linear topology is linearly compact if and only if it is complete and the factor groups modulo open subgroups satisfy the minimum condition.

Let us derive some easy consequences from Theorem $\mathrm{I}$.

COROLLARY 2. A bounded linearly compact group is compact.

A bounded group (i.e. there is an upper bound for the orders of the elements) with minimum condition is finite [2], hence by Theorem 1 the group is an inverse limit of finite groups, and therefore compact.

Recall that by the $Z$-adic topology of an abelian group $A$ is meant the (not necessarily Hausdorff) linear topology where $n A(n=1,2, \cdots)$ form a fundamental system of neighborhoods of 0 .

CoROLlaRY 3. Linear compactness in the Z-adic topology implies compactness.

Now the groups $A / n A(n=1,2, \cdots)$ are discrete, bounded and linearly compact, hence finite. Thus $A$ is an inverse limit of finite groups, and so compact.

The following examples for linearly compact groups are fundamental.

1. The group $J_{p}$ of $p$-adic integers is compact in its $p$-adic topology (this is the same as the $Z$-adic topology in this case), hence $J_{p}$ is linearly compact.

2. The group $K$ of real numbers $\bmod 1$ is, algebraically, the direct product $\Pi_{p} Z\left(p^{\infty}\right)$ with $p$ running over all primes. If each $Z\left(p^{\infty}\right)$ is equipped with the discrete topology, and then $K$ with the product topology, a linearly compact topology arises. In the sequel we consider $K$ in this topology (which is, however, not the only linearly compact topology $K$ has).

3. The group $R$ of reals has infinitely many linear topologies. In fact, $R$ is isomorphic to the additive group $K_{p}$ of all $p$-adic numbers (both are $\aleph_{0}{ }^{\text {th }}$ powers of $Q$ ), and this latter group is the inverse limit of groups $Z\left(p^{\infty}\right)$, namely, $\lim _{\leftarrow} C_{n}$ with $n=1,2, \cdots$ where the homomorphism. 
$C_{n+1} \rightarrow C_{n}$ maps elements of order $p^{k}$ upon elements of order $p^{k-1}$; $C_{n} \cong Z\left(p^{\infty}\right)$.

The following two theorems describe the relationship between compact, linearly and algebraically compact groups.

THEOREM 2. A group which admits a compact topology admits a linearly compact topology too.

It is known (see [7], [8]) that $A$ admits a compact topology exactly if it is a direct product of groups of type $Z\left(p^{n}\right), Z\left(p^{\infty}\right), J_{p}$ and $R$ where the number of groups $Z\left(p^{\infty}\right)$, for any $p$, does not exceed that of $R$. All these groups admit linearly compact topologies, so by (c) the assertion follows.

The group $Z\left(p^{\infty}\right)$ shows that a linearly compact group does not necessarily admit a compact topology.

Recall that algebraically compact groups $A$ can be defined in various equivalent ways [6] of which we mention the following ones: (i) $A$ is a direct summand (in the pure algebraic sense) of a compact group; (ii) $A$ is a direct summand in every group containing it as a pure subgroup ( $A$ is pure in $G$ if $n A=A \cap n G$ for $n=1,2, \cdots)$; (iii) if

$$
\sum_{j} n_{i j} x_{j}=a_{i}(\in A)
$$

(almost all integers $n_{i j}$ with fixed $i$ are 0 ) is a system of equations with unknowns $x_{j}$ every finite subsystem of which has a solution in $A$, then the whole system is solvable in $A$. The structure of algebraically compact groups can completely be described [11].

THEOREM 3. Every linearly compact group is algebraically compact.

The proof is the same as for compact groups ([14] or [6]). We assume $A$ linearly compact and prove that $A$ satisfies condition (iii). A solution of the $i^{\text {th }}$ equation in (1) can be regarded as an element $\left(\cdots, c_{j}, \cdots\right)$ of the product $\Pi A=A^{*}$ (with one component for every $j$ ) such that $x_{j}=c_{j}$ satisfy the $i^{\text {th }}$ equation. The set $S_{i}$ of all solutions of the $i^{\text {th }}$ equation is easily seen to be a coset mod some closed subgroup of the linearly compact group $A^{*}$. The hypothesis that finite subsystems of (1) are solvable amounts to the finite intersection property for the $S_{i}$. By linear compactness, the intersection of all the $S_{i}$ is not empty, yielding a solution for the whole system (1).

Notice that there are algebraically compact groups which cannot be linearly compact. For instance, the direct sum of countably many cyclic groups of the same order $p^{n}$ is algebraically compact, but never linearly compact (cf. Corollary 2). The same is true for the direct sum of a finite or countably infinite number of $Q$.

It is worth while mentioning that Theorem 3 is a simple consequence of Theorem 5 whose proof is, however, based on a deep duality theory. 


\section{4}

We know from Theorem 1 that every linearly compact group $A$ can be written in the form $A=\lim _{\leftarrow} M_{i}$ where the $M_{i}$ satisfy the minimum condition. By a theorem of Kurosh (see e.g. [2]) each $M_{i}$ is the direct sum of a finite number of groups $Z\left(p^{n}\right)$ with $n \leqq \infty$. If, for a prime $p$, we restrict ourselves to the $p$-components of the $M_{i}$ and to the corresponding restrictions of the homomorphisms $M_{i} \rightarrow M_{j}$, then the inverse limit of this inverse system is a group $A_{p}$ which is obviously a closed direct summand of $A$. Since $p$-groups are, in a natural way, $p$-adic modules, it follows at once that $A_{p}$ too is a $p$-adic module, moreover, it is a topological $p$-adic module, i.e. multiplications by $p$-adic integers are continuous in $A_{p}$. It is now straightforward to check that $A$ is the direct product of the $A_{p}$ with $p$ running over all primes, and the definition of topology on inverse limits shows that $A=\Pi_{p} A_{p}$ will carry the product topology.

The direct product $Z=\prod_{p} Z_{p}$ of the compact rings $Z_{p}$ of the $p$-adic integers is the completion of the ring of integers in the $Z$-adic topology; a fundamental system of neighborhoods of 0 is formed by the ideals $n Z$ $(n=1,2, \cdots)$. Every torsion group is a $Z$-module in the natural way, and it follows in the same way as in the preceding paragraph that every linearly compact group is a topological 2 -module, namely, if $A=\Pi_{p} A_{p}$ then on $A_{p}$ the $p$-adic integers act in the natural way while the $q$-adic integers $(q \neq p)$ act trivially. (For the first part of the next result cf. Schöneborn $[15]$.

THEOREM 4. Every linearly compact group $A$ is a topological Z-module, and there is a (unique) decomposition $A=\Pi_{p} A_{p}$ where, for each prime $p$, $A_{p}$ is a topological $p$-adic module.

In view of this theorem, in order to find a structure theorem on linearly compact groups, it suffices to consider only those which are at the same time topological $p$-adic modules. Kaplansky [10] established a duality theory between the classes of all linearly compact $p$-adic modules and all discrete $p$-adic modules, the characters being continuous homomorphisms into the discrete module $Z\left(p^{\infty}\right)$; cf. also Schöneborn [15]. Consequently, the linearly compact $p$-adic modules are - from the pure algebraic point of view nothing else than the groups $\operatorname{Hom}_{Z_{p}}\left(M, Z\left(p^{\infty}\right)\right)$ with discrete $p$-adic modules $M$.

To describe the Homs in question, let us start with a basic submodule $B$ of $M$; this is a direct sum of cyclic $p$-adic modules:

$$
B \cong \underset{n=1}{\oplus} \underset{\mathbf{m}_{n}}{\oplus} Z\left(p^{n}\right) \oplus \underset{\mathbf{m}_{0}}{\oplus} J_{p} \quad \text { (with cardinals } \mathfrak{m}_{n}, \mathfrak{m}_{0} \text { ) }
$$

such that $B$ is pure in $M$ and $M / B$ is divisible [2]. Thus 


$$
M / B \cong \underset{\mathbf{m}_{\infty}}{\oplus} Z\left(p^{\infty}\right) \oplus \underset{\mathbf{m}}{\oplus} K_{\boldsymbol{p}} .
$$

$Z\left(p^{\infty}\right)$ is an injective $Z_{p}$-module, thus the exact sequence

$$
0 \rightarrow B \rightarrow M \rightarrow M / B \rightarrow 0
$$

is carried by Hom $\left(*, Z\left(p^{\infty}\right)\right)$ into the exact sequence

$$
0 \rightarrow \operatorname{Hom}\left(M / B, Z\left(p^{\infty}\right)\right) \rightarrow \operatorname{Hom}\left(M, Z\left(p^{\infty}\right)\right) \rightarrow \operatorname{Hom}\left(B, Z\left(p^{\infty}\right)\right) \rightarrow 0 .
$$

(We dropped the reference to $Z_{p}$.) Since purity is preserved under Hom [4] and $\operatorname{Hom}\left(M / B, Z\left(p^{\infty}\right)\right)$ is algebraically compact as a $Z_{p}$-module too, we obtain by property (ii)

$$
\begin{aligned}
\operatorname{Hom}\left(M, Z\left(p^{\infty}\right)\right) & \cong \operatorname{Hom}\left(B, Z\left(p^{\infty}\right)\right) \oplus \operatorname{Hom}\left(M / B, Z\left(p^{\infty}\right)\right) \\
& \cong \prod_{n=1}^{\infty} \prod_{\mathbf{m}_{\mathfrak{n}}} Z\left(p^{n}\right) \oplus \prod_{\mathbf{m}_{\mathbf{0}}} Z\left(p^{\infty}\right) \oplus \prod_{\mathbf{m}_{\infty}} J_{\mathfrak{p}} \oplus \prod_{\mathfrak{m}} K_{p} .
\end{aligned}
$$

Because of $K_{p} \cong R$ we arrive at the necessity part of

THEOREM 5. A group admits a linearly compact topology if and only if it is the direct product of groups of the following types: $Z\left(p^{n}\right), Z\left(p^{\infty}\right), J_{p}, R$.

Since each of these groups does admit a linearly compact topology, the sufficiency is evident.

A comparison of the structures of compact and linearly compact groups shows that the only difference is that the inequality for the numbers of components in case of compact groups is dropped for linearly compact groups.

The cardinal invariants of a linearly compact group $A$ which form a complete system of invariants for $A$ qua algebraically compact group can be determined easily from Theorem $\mathbf{5}$.

\section{5}

Let us mention a few consequences of Theorem $\mathbf{5}$. It is known that the cardinal number of an infinite compact group is of the form $2^{m}$ with $m$ an infinite cardinal [9]. This is not true for linearly compact groups, as is shown by the group $Z\left(p^{\infty}\right)$. However, we have:

CoRollary 4. An infinite linearly compact group is either countable or is of power $2^{\mathrm{m}}$ for some infinite cardinal $\mathrm{m}$.

Notice that if $A$ is the direct product of $\mathfrak{m} \geqq \aleph_{0}$ groups, each of type $Z\left(p^{n}\right), Z\left(p^{\infty}\right), J_{p}$ or $R$, then $A$ is of cardinality $2^{m}$.

It follows at once that the linearly compact groups of cardinality $\aleph_{0}$ are just the finite direct sums of groups $Z\left(p^{n}\right)$ with $n \leqq \infty$, thus 
Corollary 5. A linearly compact group is countable if and only if it satisfies the minimum condition (i.e. it is discrete).

Next we wish to find the cardinal number of the set of non-isomorphic linearly compact groups of a given cardinality $\mathfrak{n}$. If $\mathfrak{n}=\boldsymbol{\aleph}_{0}$, then Corollary 5 implies that the cardinal number in question is $\boldsymbol{\aleph}_{0}$. If $\mathfrak{n}=2^{\mathfrak{m}}\left(\mathfrak{m} \geqq \boldsymbol{N}_{0}\right)$, then we argue as follows. If $M$ is a discrete $p$-adic module generated by $\mathrm{m}$ elements, then (2) shows that $\operatorname{Hom}_{Z_{p}}\left(M, Z\left(p^{\infty}\right)\right)$ is of cardinality $2^{\mathfrak{m}}$. If $M$ is the direct sum of discrete $p$-adic modules, one for each prime $p$, then $\operatorname{Hom} \mathcal{Z}(M, K)=A$ is of cardinality $2^{\mathfrak{m}}, \mathfrak{m}$ denoting the number of generators of $M$. Since every linearly compact group $A$ arises in this way, it is clear that the cardinal number of the set of linearly compact groups of power $\leqq 2^{\mathrm{m}}$ is equal to the cardinal number of the set of direct sums of $p$-adic modules generated by $\leqq \mathfrak{m}$ elements. For $\mathfrak{m}=\boldsymbol{\aleph}_{0}$ this number is $2^{\aleph_{0}}$, while for $m \geqq 2^{\aleph_{0}}$ this number is obviously $\leqq 2^{m}$. But there exist $2^{m}$ non-isomorphic $p$-groups of cardinality $\mathfrak{m}$ (see [2] or [3]), so we conclude:

COROLlaRY 6. For each infinite cardinal $\mathfrak{m}$, the set of all non-isomorphic linearly compact groups of power $2^{\mathbf{m}}$ is of power $2^{\mathbf{m}}$.

Moreover, one has a much finer result:

COROLLARY 7. There exist $2^{\mathrm{m}}$ non-isomorphic linearly compact groups of power $2^{\mathrm{m}}$ which are algebraically isomorphic.

This is the same as Corollary 7 in [3].

This result should be compared with the fact that there are groups which admit exactly one linearly compact topology. For instance, if the group $A$ is the direct product of $\mathfrak{m}$ copies of $Z\left(p^{n}\right)$ (with fixed integer $p^{n}$ ) or of $J_{p}$ (with fixed $p$ ), then $A$ cannot be anything else than the character group of the direct sum of $m$ copies of $Z\left(p^{n}\right)$ and $Z\left(p^{\infty}\right)$, respectively, so it can carry a single linearly compact (or compact) topology (which is easily seen to be the topology defined by all subgroups of finite index). [In the last argument the generalized continuum hypothesis was assumed.]

\section{6}

Finally, we mention an interesting result on linear compactness.

We have seen that the group of $Z_{p^{-}}$-homomorphisms of discrete $Z_{p^{-}}$ modules into $Z\left(p^{\infty}\right)$ are linearly compact. A more general result can be established:

TheOREM 6. If $A$ is a linearly compact group, then both Hom $(G, A)$ and $\operatorname{Hom}_{Z}(M, A)$ are linearly compact for any group $G$ and 2 -module $M$.

The functor $\operatorname{Hom}_{S}(X, *)$ (for any ring $S$ ) commutes with inverse limits $[1]$, therefore by (d) and Theorem 1 we need only consider the case 
when $A$ satisfies the minimum condition, moreover, when $A \cong Z\left(p^{n}\right)$ for $n \leqq \infty$. The case $n=\infty$ is settled by the proof of Theorem 5 for $Z$-modules, while for groups $G$ a similar argument applies with basic submodules replaced by $p$-basic subgroups [5]. For $n<\infty$, both $G$ and $M$ can be replaced by $G / p^{n} G$ and $M / p^{n} M$, which are direct sums of groups of orders $p^{k}(k \leqq n)$. Thus Hom $\left(*, Z\left(p^{n}\right)\right)$ will be the direct product of the same groups, and hence linearly compact.

Notice that the linearly compact topologies on the groups Hom $\left(*, Z\left(p^{n}\right)\right)$ induce a linearly compact topology on the group of homomorphisms into $A$.

\section{References}

[1] H. Cartan and S. Eilenberg, Homological algebra (Princeton, 1956).

[2] L. Fuchs, Abelian groups (Budapest, 1958).

[3] L. Fuchs, 'On character groups of discrete abelian groups', Acta Math. Acad. Sci. Hung. 10 (1959), $133-140$.

[4] L. Fuchs, 'Notes on abelian groups I', Annales Univ. Sci. Budapest 2 (1959), 5-23.

[5] L. Fuchs, 'Notes on abelian groups II', Acta Math. Acad. Sci. Hung. 11 (1960), 117-125.

[6] L. Fuchs, 'On algebraically compact abelian groups', J. Nat. Sci. and Math. 3 (1963), $73-82$.

[7] D. K. Harrison, 'Infinite abelian groups and homological methods', Annals of Math. 69 (1959), 366-391.

[8] A. Hulanicki, 'Algebraic structure of compact abelian groups', Bull. Acad. Polon. Sci. $6(1958), 71-73$.

[9] S. Kakutani, 'On cardinal numbers related with a compact abelian group', Proc. Imp. Acad. Tokyo 19 (1943), 366-372.

[10] I. Kaplansky, 'Dual modules over a valuation ring', Proc. Amer. Math. Soc. 4 (1953), $213-219$.

[11] I. Kaplansky, Infinite abelian groups (Ann Arbor, 1954).

[12] S. Lefschetz, Algebraic topology (New York, 1942).

[13] H. Leptin, 'U'ber eine Klasse linear kompakter abelscher Gruppen I', Abh. Math. Sem. Univ. Hamburg 19 (1954), 23-40.

[14] J. Łoś, 'Abelian groups that are direct summands of every abelian group which contains them as pure subgroups', Fundamenta Math. 44 (1957), 84-90.

[15] H. Schöneborn, 'Ủber gewisse Topologien in Abelschen Gruppen I', Math. Z. 59 (1954), $455-473$; 'II', 60 (1954), $17-30$.

[16] S. M. Yahya, ' $P$-pure exact sequences and the group of $p$-pure extensions', Annales Univ. Sci. Budapest 5 (1962), 179-191.

University of Miami

Coral Gables, Florida, U.S.A. 